\title{
Effect of weak attractive interactions on flow behavior of highly concentrated crystalline suspensions
}

\author{
Clara Weis, ${ }^{\text {a) }}$ Irene Natalia, and Norbert Willenbacher \\ Institute for Mechanical Process Engineering and Mechanics, Applied Mechanics, \\ Karlsruhe Institute for Technology (KIT), Karlsruhe, Germany
}

(Received 31 December 2013; final revision received 30 May 2014;

published 27 August 2014)

\begin{abstract}
Synopsis
Weak attractive interactions lead to a substantial broadening of the fluid-crystalline coexistence region of crystalline, colloidal hard-sphere dispersions. Here, we demonstrate that a weak depletion attraction introduced by nonadsorbing polymer added to the continuous phase results in a strong decrease of the low shear viscosity even at particle concentrations up to $\Phi=0.65$ analogous to the re-entry phenomenon in highly concentrated glassy systems. This promotes flow in macroscopic channels but the scenario changes in confined, microfluidic geometries. Pure crystalline suspensions exhibit flow induced melting in the region of highest shear rates close to the wall, and this promotes flow through microchannels $(<100 \mu \mathrm{m})$. In contrast, weak attractive interactions fortify preferential crystallization at the channel walls finally resulting in channel clogging and cessation of flow. This wall crystallization phenomenon particularly impairs viscosity determination from classical parallel plate rotational rheometry if the gap width is set too low. These findings may find application in the important biotechnological field of protein preparation and crystallization. (C) 2014 The Society of Rheology. [http://dx.doi.org/10.1122/1.4882955]
\end{abstract}

\section{INTRODUCTION}

Phase behavior and structure of colloidal dispersions are of interest because they are widely used in fabrication of nanostructured materials. They also serve as model systems for studying fundamental physical phenomenon like crystallization or glass formation on easily accessible spatial and temporal scales [Pusey and van Megan (1986); Pusey et al. (1994); Lekkerkerker et al. (1992)]. A key technological challenge is to control the flow properties in order to meet the manifold requirements during processing and application. This is especially relevant when dealing with nanoparticle formulations on a technical scale where high particle loading is required [Willenbacher et al. (2011)]. When these suspensions are forced to flow through fine geometries, the presence of nearby walls and surfaces may induce additional interactions or wall slip [Walls et al. (2003); Kalyon (2005); Ballesta et al. (2012)], which can lead to nonuniform flows and clogging [Wyss et al. (2006)].

The classical route to achieve highly concentrated dispersions at low viscosity level is to provide a bimodal or a broad particle size distribution [Farris (1968); Dames et al.

\footnotetext{
${ }^{a)}$ Author to whom correspondence should be addressed; electronic mail: clara.weis@kit.edu
} 
(2001)], but this is not always technically or economically feasible and large particles may disturb the final product properties. An alternative concept to make highly concentrated, freely flowing dispersions is based on the introduction of weak attractive depletion interactions [Lekkerkerker et al. (1992); Poon (2002); Asakura and Oosawa (1958)]. For glass forming systems, the so-called re-entry phenomenon has been predicted theoretically [Fabbian et al. (1999a); Fabbian et al. (1999b); Dawson et al. (2001)] and confirmed experimentally for various model systems. Diffusive motion of particles is accelerated and fluid states are observed even at particle loadings beyond the colloidal glass transition $\left(\Phi_{\mathrm{g}}=0.58\right)$ due to the weak attraction among particles [Pham et al. (2002); Pham et al. (2004); Shah et al. (2003a); Shah et al. (2003b); Eckert and Bartsch (2004); Eckert and Bartsch (2002); Eckert and Bartsch (2003)]. Beyond that, even the macroscopic flow behavior changes and the low shear viscosity drops drastically [Willenbacher et al. (2011)]. The weak attractive interactions are supposed to lead to reversible particle clustering, which opens the space allowing for long-range particle motion and macroscopic flow. Attractive interactions among particles can be finely tuned via addition of nonadsorbing polymer to the continuous phase. Polymer molecules and colloidal particles are mutually impenetrable. Thus, the center of a polymer coil with the radius $R_{g}$ is excluded from a region of thickness $R_{g}$ from the surface of each particle. This area is called the "depletion region." If the depletion regions of two particles overlap, there is an unbalanced osmotic pressure pushing the particles together which leads to particle clustering.

Our goal is to transfer the fluidization concept suggested and confirmed for glassy systems to crystalline dispersions. This is of fundamental physical interest but also may have a technical impact. Crystallization is a common method for the purification of protein solutions, and depletion interactions also play an important role [Kulkarni and Zukoski (2001)]. Nonadsorbing polymers like poly (ethylene glycol) with low molecular weight are often used to modify the phase behavior of protein solutions [Hosfield et al. (2003); Annunziata et al. (2003); Galkin and Vekilov (2000)]. On the other hand, Valadez-Pérez et al. (2012) have investigated the phase behavior of colloidal suspensions with short-range attractive interactions using theoretical concepts as well as Monte Carlo simulation methods. They compare their results with experimental data for suspensions of the globular protein lysozyme. Here, we discuss the effect of added polymer on the flow properties of crystalline colloidal polymer dispersions. Classical mechanical rheometry and a self-constructed flow channel on an inverted fluorescence microscope are used to study the flow properties both on macroscopic and microfluidic level.

Up to now, the influence of added polymer in crystalline dispersions has been studied via light scattering techniques, Bragg reflection, and simulation [Ilett et al. (1995); Poon (2002); Beyer et al. (2012); Palberg and Stipp (2009)]. The addition of a small amount of polymer leads to a broadening of the fluid-crystalline coexistence region [Ilett et al. (1995); Pusey et al. (1991)]. Gast et al. (1983) predicted that the effect of added polymer depends crucially on the polymer to particle size ratio $\xi=\mathrm{R}_{\mathrm{g}} / \mathrm{R}$. For a size ratio less than 0.25 , the added polymer merely expands the colloidal-crystal coexistence region. For size ratio larger than 0.25 , a three phase coexistence of colloidal gas, liquid, and crystal phase is observed [Pusey et al. (1994); Ilett et al. (1993)].

Simulations of glass forming systems revealed that the so-called re-entry phenomenon, i.e., the fluidization due to weak attraction vanishes for $\xi \leq 0.03$ [Zaccarelli et al. (2004)]. Mode coupling theory predicts the transition from the liquid to glass within two lines, the repulsive driven line, and the attractive driven line. For $\xi=0.03$, a discontinuity is predicted between the repulsive and the attractive driven line. The system does not 
reach an ergodic state with increasing polymer concentration; instead there is a sudden transition from the repulsive into the attractive glass.

The density of the crystal phase in the fluid-crystalline coexistence region was determined experimentally from the Bragg peaks obtained by small angle light scattering (SALS) [Stipp et al. (2010)]. The packing density of the crystals is found to be higher than the overall volume fraction $\Phi$ of the suspension since crystalline regions coexist with fluid regions of lower particle density which is also predicted theoretically [Lekkerkerker et al. (1992)]. For polymer-free samples, polycrystalline solids are obtained via homogeneous nucleation and bulk crystallization with a little competition of wall crystal formation [Beyer et al. (2012); Palberg and Stipp (2009)]. The crystallization changes upon addition of polymer. When nonadsorbing polymer is added, the precursorto-crystal transformation for the bulk crystallization is delayed and crystallization kinetics slows down, while the induction time for wall nucleation is reduced. This can be rationalized considering that the depletion attraction between a sphere and a wall is stronger than between two spheres since the overlapping volume is larger in the former case [Kaplan et al. (1994)]. At the same time, large polymer concentration suppresses bulk crystal growth and coarsening, such that after the precursor-to-crystal conversion the bulk crystals retain their size without further growth.

The investigations of colloidal crystals under shear were mostly done with a confocal microscope. Shear-induced melting and crystallization of concentrated colloidal hard-sphere dispersions were investigated using confocal microscopy in combination with a counter rotating shear cell [Imhof et al. (1994); Derks et al. (2009)]. The effect of added polymer on the flow of semiconcentrated mono- and bimodal colloidal suspensions in microchannels has also been investigated [Pandey and Conrad (2012)]. Smith et al. (2007) have shown that shear-induced crystallization can occur even in strongly attractive glasses at intermediate particle concentration around $40 \%$. The scope of the work presented here is to study the effect of weak attractive interactions induced by added polymer on the behavior of concentrated crystalline colloidal suspensions in macroscopic and microchannel flows.

\section{MATERIAL AND METHODS}

The colloidal dispersion investigated here consists of short-range repulsive poly (styrene-butyl acrylate) $\mathrm{P}(\mathrm{S} / \mathrm{BA}$ ) particles (provided by BASF $\mathrm{SE}$ ) suspended in water. Rheological measurements were performed with volume fractions $\Phi$ between 0.55 and 0.65 . For the microchannel, the chosen volume fraction was 0.52 . The particle radius is $\mathrm{R}=130 \pm 1.5 \mathrm{~nm}$. The polydispersity is 0.03 , measured by dynamic light scattering. Acrylic acid was used as a surface functional co-monomer at a weight fraction of $2 \%$, relative to the total monomer concentration. The dispersion was made using classical emulsion polymerization. The $p \mathrm{H}$ was adjusted to 7 with $\mathrm{NaOH}$ (Carl Roth), such that the acrylic acid was dissociated providing short-range electrosteric repulsion. Linear polyethylene oxide (PEO) (Merck) with molecular masses $\mathrm{M}_{\mathrm{w}}=10,20$, and $35 \mathrm{~kg} / \mathrm{mol}$ was used to introduce weak attractive depletion forces. The $\mathrm{M}_{\mathrm{w}}$ values vary from batch to batch between 9 and $12.5 \mathrm{~kg} / \mathrm{mol}$ for a specified nominal $\mathrm{M}_{\mathrm{w}}$ of $10,16-25 \mathrm{~kg} / \mathrm{mol}$ for nominal $\mathrm{M}_{\mathrm{w}}$ of 20 , and $28-38 \mathrm{~kg} / \mathrm{mol}$ for nominal $\mathrm{M}_{\mathrm{w}}$ of $35 \mathrm{~kg} / \mathrm{mol}$, according to the supplier. This corresponds to polymer to particle size ratios $\xi=\mathrm{R}_{\mathrm{g}} / \mathrm{R}=0.03 \pm 0.008$, $0.048 \pm 0.012,0.067 \pm 0.016$, respectively [Kawaguchi et al. (1997)]. PEO concentration was varied between 1 and $10 \mathrm{~g} / \mathrm{l}$. Note that the given concentrations refer to the volume of the aqueous phase. According to Asakura-Oosawa [Asakura et al. (1958)], we have estimated the depletion interaction potential of two particles in contact as 


$$
\frac{\Psi_{d e p}}{k T}=-\frac{3}{2}\left(\frac{R}{R_{g}}\right) \Phi_{p},
$$

where $\Phi_{\mathrm{p}}$ is the volume concentration of the polymer in the liquid phase which is estimated as

$$
\Phi_{p}=\frac{4 \pi}{3} R_{g}^{3} \frac{N}{V},
$$

where $\mathrm{N}$ denotes the total number of polymer molecules in solution and $\mathrm{V}$ is the total volume of the liquid phase. Note that this simple Eq. (1) is valid for dilute suspensions and serves as a rough estimate here. At the high particle loadings investigated here, the true depletion interaction may be 4-5 times higher due to the so-called collective effect from the neighboring particles [Dijkstra et al. (1999)].

In addition, Table I also includes the osmotic pressure $\Pi=(N / V) R T$ calculated from the molecular weight and concentration of the dissolved polymer.

The samples were prepared via two dialysis steps. First, the suspension was dialyzed against $10 \mathrm{mM} \mathrm{NaCl}$ solution in order to remove oligomers from the serum. Therefore, a dialysis membrane (Carl Roth) with a molecular weight cut off (MWCO) of $14 \mathrm{~kg} / \mathrm{mol}$ was used. After that a dialysis membrane (Carl Roth GmbH, MWCO: 4000-6000 g $\mathrm{mol}^{-1}$ ) filled with the dispersion and the calculated amount of polymer was immersed in a dialysis bath filled with an aqueous solution of $35 \mathrm{~kg} / \mathrm{mol}$ PEO at a concentration of $15 \%$ by weight. The $p \mathrm{H}$ of this solution was also adjusted to 7 . The high osmotic pressure of the PEO solution concentrates the dispersion to the desired particle volume fraction $\Phi$.

Classical methods to determine the volume fraction for hard spheres via the crystalfluid coexistence [Segré et al. (1995); Pusey and van Megan (1986)] did not work, as heterogeneous crystallization [Poon (2002)], indicated by the opalescence at the wall, dominated. Therefore, we determined the volume fraction $\Phi$ of our samples as follows: First, we measured the solid content $x_{P S / B A}$ of the sample using a gravimetric halogen moisture analyzer (HG83, Mettler Toledo). After that we measured the density $\rho_{\text {total }}$ of the whole sample with a pycnometer (Carl Roth). We calculated the density $\rho_{P S / B A}$ of the particles, using Eq. (1), where $x_{w}$ is the mass fraction and $\rho_{w}$ is the density of water.

TABLE I. Osmotic pressure $\Pi$ and two particle depletion interaction energy $\Psi_{\text {dep }} / \mathrm{kT}$ according to the Asakura-Oosawa model estimated for different molecular masses and polymer concentration.

\begin{tabular}{lccc}
\hline \hline $\mathrm{M}_{\mathrm{w}}(\mathrm{g} / \mathrm{mol})$ & $\mathrm{c}_{\mathrm{p}}(\mathrm{g} / \mathrm{l})$ & $\Pi(\mathrm{Pa})$ & $-\Psi_{\text {dep }}(\mathrm{kT})$ \\
\hline 10000 & 1 & 0.24 & 0.86 \\
& 3 & 0.74 & 2.60 \\
& 8 & 1.97 & 6.94 \\
20000 & 10 & 2.46 & 8.67 \\
& 1 & 0.12 & 0.97 \\
& 3 & 0.37 & 2.92 \\
35000 & 8 & 0.98 & 7.80 \\
& 10 & 1.23 & 9.76 \\
& 1 & 0.07 & 1.06 \\
& 3 & 0.21 & 3.19 \\
& 8 & 0.56 & 8.50 \\
\hline \hline
\end{tabular}




$$
\rho_{P S / B A}=\frac{x_{P S / B A}}{\left(\frac{1}{\rho_{\text {total }}}-\frac{x_{w}}{\rho_{w}}\right)}
$$

The volume fraction $\Phi$ is then given by

$$
\Phi=\frac{x_{P S / B A} * \rho_{\text {total }}}{\rho_{P S / B A}} .
$$

In order to estimate the range of the repulsive interaction, the Debye length was estimated from the measured conductivity of the serum after the first dialysis and the known concentration dependence of the conductivity for $\mathrm{NaCl}$ solutions. This results in a Debye length $\kappa=1.7 \pm 0.3 \mathrm{~nm}$.

\section{A. Rheological characterization}

Steady and oscillatory shear measurements were performed using a stress-controlled Physica MCR 501 (Anton Paar). Depending on the volume fraction, different cone-plate geometries were used with different radius $(8-35 \mathrm{~mm}$ diameter). In steady shear rate measurements, the shear rate was varied between 0.01 and $1000 \mathrm{~s}^{-1}$. Oscillatory shear experiments covered the frequency range from 0.01 to $100 \mathrm{rad} \mathrm{s}^{-1}$. Amplitude sweep measurements were performed in advance to determine the allowed strain for probing the linear viscoelastic fluid properties. Prior to all measurements, a preshear test was carried out to ensure a well-defined initial condition. The preshear test consist of 2 min waiting time and subsequent $500 \mathrm{~s}$ of oscillatory preshearing with a frequency of $6.3 \mathrm{rad} \mathrm{s}^{-1}$ and a deformation $\gamma$ of $4.5 \%$ as already described in [Willenbacher et al. (2011)]. Plate-plate geometry (diameter $25 \mathrm{~mm}$ ) with gap heights between 0.1 and $2 \mathrm{~mm}$ was used to investigate the effect of wall slip and thixotropy. After 2 min of waiting time, the shear rate was increased from 0.1 to $1000 \mathrm{~s}^{-1}$ and back. This procedure was repeated for different periods of time. The temperature was set at $20 \pm 0.1^{\circ} \mathrm{C}$ using a piezoelectric heating device.

\section{B. Flow channel}

A self-built flow cell was used to determine flow profiles of the dispersion on the micro scale. The cross section of this microchannel is $30 \times 800 \mu \mathrm{m}$, and its length is $58.8 \mathrm{~mm}$. Microchannels with a cross section of $200 \times 200 \mu \mathrm{m}$ were used too, but since the samples are opaque determination of the full flow profile from tracer particle velocimetry was not feasible. For the samples investigated here tracking of fluorescent tracer particles was possible within a penetration depth of about $30 \mu \mathrm{m}$. The microchannel chips (microfluidic chipshop) are made from polystyrene, the cover lid is topaz with a thickness of $140 \mu \mathrm{m}$. A syringe pump (neMESYS Cetoni) combined with a $250 \mu \mathrm{l}$ syringe (ILS) was used to provide flow rates between 0.00025 and $0.001 \mu \mathrm{l} \mathrm{s}^{-1}$ corresponding to shear rates between 5 and $20 \mathrm{~s}^{-1}$. Fluorescent tracer particles (Bang laboratories) with $\mathrm{R}=140 \mathrm{~nm}$ were added to the dispersion at a concentration of $0.1 \%$ vol. in order to visualize the flow profile. These tracer particles were detected using the inverse fluorescence microscope Zeiss Axio Observer D1. The used objective is an Optifluar $100 \mathrm{x}$ with a N.A. of 1.4 (Zeiss) with a working distance of $170 \mu \mathrm{m}$ and a depth of field of $1 \mu \mathrm{m}$. A high-speed sCMOS camera (Andor Zyla) with a frame rate of $100 \mathrm{fps}$ was used to track the particles. The flow profile was determined based on the analysis of spatially resolved particle velocity in different planes perpendicular to the optical axis (z-axis in Fig. 1). The narrowest dimension of the flow channel was oriented parallel to the optical axis of 
the microscope, and a so-called z-scan device (PI Instrumente) was used to obtain the flow profile in this direction perpendicular to the flow [z-y plane in Fig. 1(b)]. This device is placed between the microscope revolver and the lens. It has a travel range of $400 \mu \mathrm{m}$ at a repetition accuracy of $5 \mathrm{~nm}$.

For each position of the $z$-scan, we took 1000 frames in $10 \mathrm{~s}$ in order to determine the velocity within one layer. Thus the velocity of approximately 20 particles in the focal plane (depth $\approx 1 \mu \mathrm{m}$ ) was determined simultaneously. The pictures were processed with the filters gauss $5 \times 5$, locally adaptive background detection and clipping $1 \%$ using the image processing software IPS (visiometrics). First step for determination of the velocity is the identification of the center of mass of each particle in each frame. From the displacement of each particle between at least 100 frames and the corresponding timelag, the velocity of each particle could be obtained. This step was performed with the commercial software Trace (visiometrics). The corresponding average value of all particle velocities was assigned as the velocity of this layer. This way the flow profile in the direction of highest shear was obtained layer by layer with a spatial resolution of $2 \mu \mathrm{m}$.

Additionally, the flow was observed via the ocular of the microscope. Looking through the ocular allows an observation of the crystals moving in the focal plane. The images shown later, in this paper, were taken through the ocular of the microscope. A schematic picture of the whole setup is shown in Fig. 1.
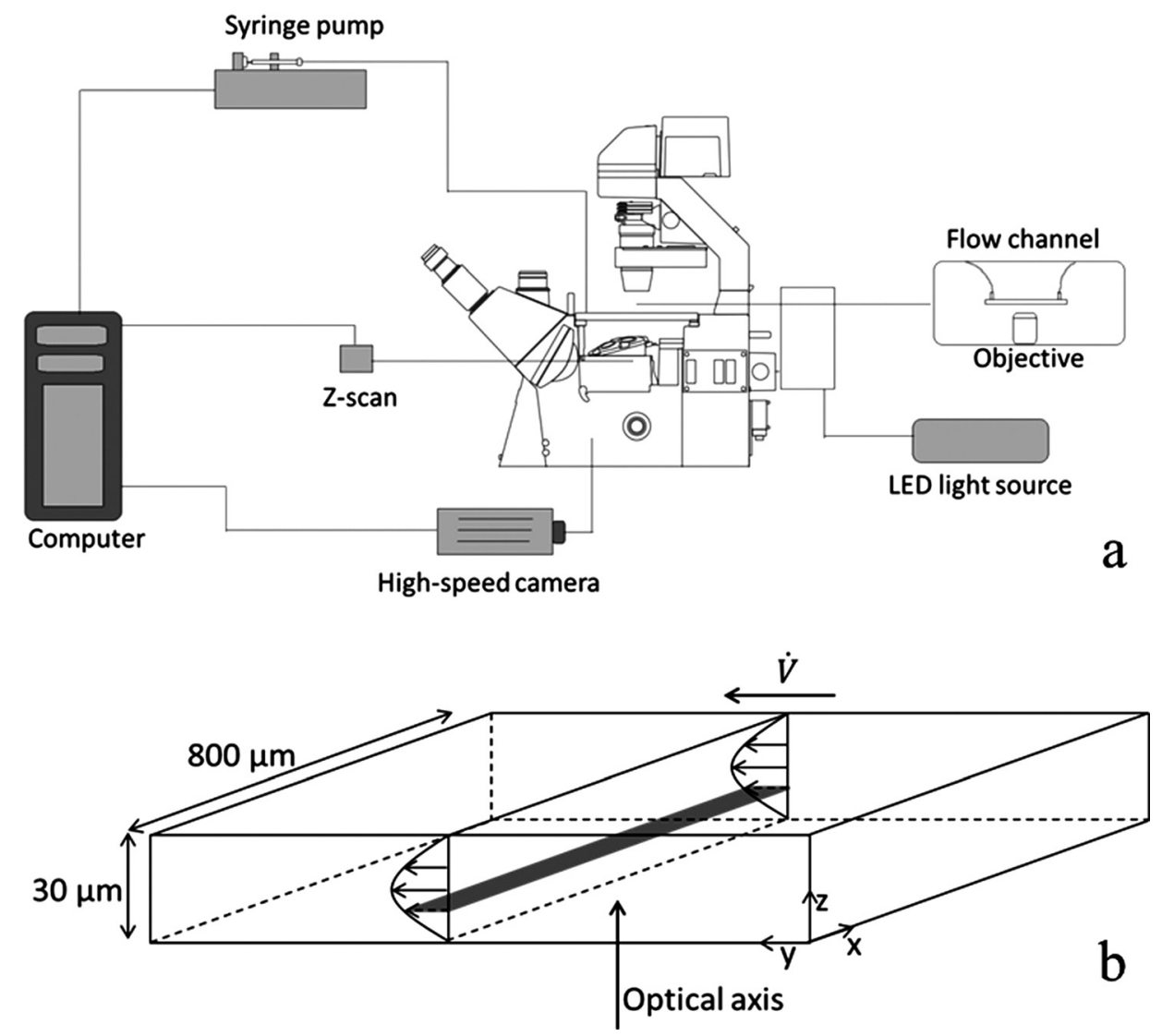

FIG. 1. (a) Schematic description of the flow cell including the syringe pump and the components of the optical fluorescent microscopy setup. The flow channel with the necessary inlet and outlet connection as well as the objective underneath is shown separately on the left. (b) Close up of the microchannel and the orientation of the optical axis. Via the ocular of the microscope one observes the $x-y$ plane, marked as the grey area. 


\section{RESULTS AND DISCUSSION}

The aqueous poly (styrene-butylacrylate) latex electrosterically stabilized by copolymerization of acrylic acid used to study the influence of added polymer on the flow of crystalline dispersions behaves almost like a hard-sphere system [Vesaratchanon et al. (2010)]. The zero-shear viscosity diverges at a critical volume fraction $\Phi_{\mathrm{g}, \mathrm{exp}}$. From the ratio of $\Phi_{\mathrm{g}, \exp }$ and the hard-sphere glass transition $\Phi_{\mathrm{g}, \mathrm{HS}}=0.58$, we have determined the effective volume fraction $\Phi_{\text {eff }}=\Phi\left(\Phi_{g, \exp } / \Phi_{g, H S}\right)$ which corresponds to a thickness of the stabilizing hairy surface layer of about $5 \mathrm{~nm}$ at $p \mathrm{H}=7$. This calculation is based on $\eta_{0}$-data for volume fractions $\Phi$ of $0.05,0.1,0.3$, and 0.4 at which the dispersion does not crystallize and thus is just a rough estimate. Presumably, the hairy layer is less extended at the high volume fractions investigated here since the ionic strength increases with increasing $\Phi$. Furthermore, the added PEO may affect the thickness of the hairy layer although the osmotic pressure induced by the added polymer is very low (cf. Table I), but a quantitative prediction is not possible here since we do not know the exact copolymer composition and the monomer concentration in the hairy layer. As a consequence, we will subsequently not consider the effective volume fraction including the hairy layer but the nominal volume fraction calculated from the weight and density of the suspended particles.

For volume fractions $\Phi>0.4$, opalescent structures are visible at the cuvette wall. After some weeks little crystals settle down at the container bottom due to gravity.

PEO has been used as a nonadsorbing depletion agent, and the fluidization is directly visible in the images of the cuvettes containing dispersions with fixed particle volume fraction $\Phi=0.58$ but varying polymer concentration $c_{p}$ shown in Fig. 2 . The surface of the sample without polymer is uneven since leveling is suppressed by the high viscosity. With added polymer a meniscus forms up to a PEO concentration of $8 \mathrm{~g} / \mathrm{l}$ of PEO. Further increasing the amount of PEO leads to gelation, i.e., formation of a strong attractive glass. Accordingly, the wall of the cuvette is not completely wetted with the dispersion, the sample surface is uneven and the opalescent appearance vanishes at a PEO concentration of $10 \mathrm{~g} / \mathrm{l}$. In addition, the expansion of the fluid-crystalline coexistence due to the added polymer can be seen. The sample without added PEO is crystalline from the bottom to the middle of the cuvette, whereas the samples with $c_{p}$ between 1 and $8 \mathrm{~g} / \mathrm{l}$ are fully crystalline. Figure 2 refers to $\Phi=0.58$, but similar behavior has been observed for other volume fractions up to 0.65 . For samples with volume fractions up to $\Phi=0.58$

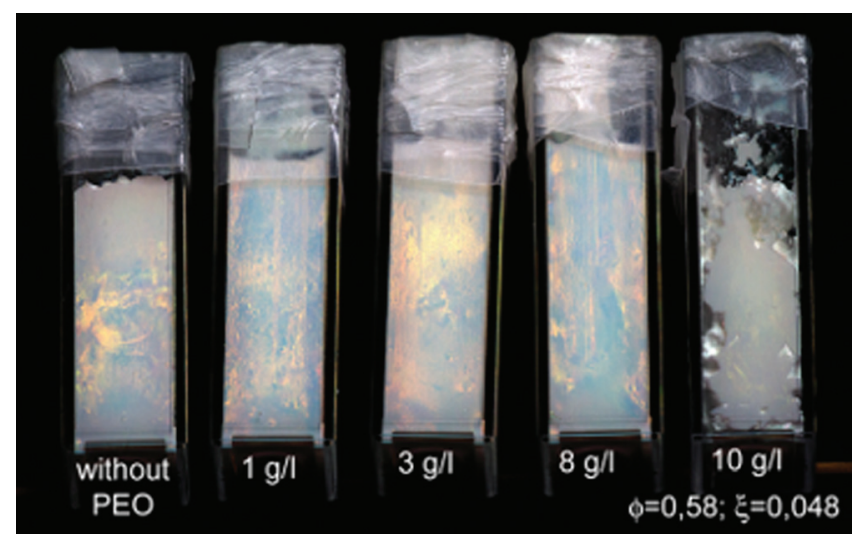

FIG. 2. Texture and optical appearance of the $\mathrm{P}(\mathrm{S} / \mathrm{BA})$ dispersion without added $\mathrm{PEO}$ (left) and with added PEO $\left(\mathrm{M}_{\mathrm{w}}=20 \mathrm{~kg} / \mathrm{mol}\right), \mathrm{c}_{\mathrm{p}}=1,3,8$, and $10 \mathrm{~g} / \mathrm{l}$ (from the left to the right) at $\Phi \approx 0.58$. 
opalescent structures occurred immediately after filling of the cuvette for PEO concentrations up to $8 \mathrm{~g} / \mathrm{l}$. For samples with higher particle loading and higher polymer concentrations, it took hours to weeks for the structure to appear.

The sample with $\Phi \approx 0.58$ and no added PEO shown in Fig. 2 is not fully crystalline yet as expected for an ideal monodisperse hard-sphere suspension. This is presumably due to the polydispersity of the particle size distribution [Sollich and Wilding (2011)]. Furthermore, the sample may not have reached equilibrium when the image was taken 1 week after filling the cuvette.

\section{A. Macroscopic flow behavior}

Molecular mass of the PEO added to the dispersions varied between $10 \mathrm{~kg} / \mathrm{mol}$ and $35 \mathrm{~kg} / \mathrm{mol}$ corresponding to $\xi=\mathrm{R}_{\mathrm{g}} / \mathrm{R}$ a $=0.03 .0 .048$ and 0.067 . For $\xi=0.03$, the samples were not homogeneous, particles formed clusters which could not be redispersed by adding water. In the following, we will therefore focus on the systems with $\xi=0.048$ and 0.067 . Fluid states were observed at polymer concentrations $c_{p}=1,3$ and $8 \mathrm{~g} / \mathrm{l}$ but not at higher polymer concentration. The change in the macroscopic flow behavior with $c_{p}$ is shown in Fig. 3(a) for a particle volume fraction $\Phi=0.55$ and $M_{w}=20 \mathrm{~kg} / \mathrm{mol}$. Similar to glass forming systems the introduction of weak depletion attraction leads to a sharp drop in the low shear viscosity by almost two orders of magnitude, whereas the high shear viscosity $\left(\dot{\gamma}>100 \mathrm{~s}^{-1}\right)$ is independent of the added polymer. The hump observed in the viscosity curves at shear rates between 1 and $10 \mathrm{~s}^{-1}$ and polymer concentrations of $8 \mathrm{~g} / \mathrm{l}$ or even more pronounced at $3 \mathrm{~g} / \mathrm{l}$ indicates a characteristic structural change which may be related to a change of crystal size and orientation. Preliminary experiments switching between periods of small amplitude oscillatory shear and steady shear rates below and above the hump are shown in Fig. 3(b). Since the moduli stay essentially at a constant level even after extended periods of high shear there is no indication of a change in crystal structure. There is also no indication for other thixotropic effects since the viscosity remains constant at this period of high shear. Further investigations regarding this rheological and structural feature are beyond the scope of this work.
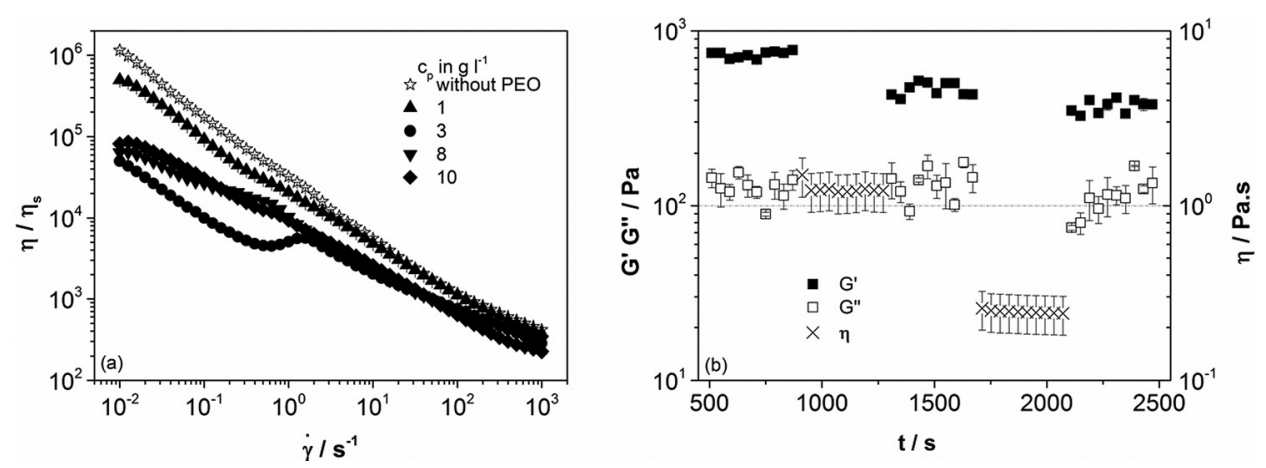

FIG. 3. (a) Relative viscosity versus shear rate for a series of acrylate dispersions with similar volume fraction $\Phi \approx 0.55$ but different PEO concentrations $\left(\mathrm{M}_{\mathrm{w}}=20 \mathrm{~kg} / \mathrm{mol}\right)$ as indicated in the legend. (b) Results from alternating oscillatory shear and steady shear measurements $\left(\Phi \approx 0.55\right.$ and $\left.\mathrm{c}_{\mathrm{p}}=3 \mathrm{~g} / \mathrm{l}\right)$. The measurement protocol was as follows: 1 . Waiting time $500 \mathrm{~s}$ at rest. 2 . Oscillatory shear within the linear viscoelastic response regime (LVR) for $500 \mathrm{~s}$ at $\gamma=0.8 \%$ and $\omega=100 \mathrm{rad} \mathrm{s}^{-1}$. LVR was confirmed by preliminary amplitude sweeps. 3. Steady shear at $\sigma=0.8 \mathrm{~Pa}$ for $500 \mathrm{~s}$ (which is below the hump of the viscosity). 4. Small amplitude oscillatory shear for $500 \mathrm{~s}$ similar as 2. 5. Steady shear for $500 \mathrm{~s}$ at $\sigma=60 \mathrm{~Pa}$. 6 . Small amplitude oscillatory shear for $500 \mathrm{~s}$ similar as 2. Measurements were performed using a cone-plate geometry with gap height of $1.3 \mathrm{~mm}$ at the rim. 
The $\mathrm{c}_{\mathrm{p}}$-dependence of the low shear viscosity at a fixed shear rate is displayed in Fig. 4(a) for fixed $\xi=0.048$ and different $\Phi$ clearly demonstrating a pronounced viscosity minimum at a polymer concentration between 3 and $8 \mathrm{~g} / \mathrm{l}$. As slipping [Ballesta et al. (2012)] or shear melting [Imhof et al. (1994)] may occur and influence the apparent viscosity curve, small amplitude oscillatory experiments were performed in addition. In Fig. 4(b), $\mathrm{G}^{\prime}$ data obtained at $\omega=1 \mathrm{rad} \mathrm{s}^{-1}$ are displayed as a function of polymer concentration. Both $\mathrm{G}^{\prime}$ and the low shear viscosity show the same trend and exhibit minimum values at intermediate polymer concentrations between 3 and $8 \mathrm{~g} / \mathrm{l}$. Similar results were found for $\mathrm{G}^{\prime \prime}$ but for sake of clarity data are not shown here. Finally, we conclude that the minimum in low shear viscosity is not an artifact from shear melting and slip effects, but a true material property. This phenomenon is attributed to the broadening of the fluid-crystalline coexistence region due to weak attractive interactions among particles as reported for colloidal hard-sphere dispersions [Ilett et al. (1995)]. Due to this attraction, the fluid phase gets more dilute while the crystals are packed denser and this seems to facilitate flow finally resulting in the observed strong decrease of low shear viscosity.

Figure 5 shows the variation of the low shear viscosity as a function of polymer concentration for $\Phi=0.58$ but two size ratios $\xi=0.048$ and $\xi=0.065$. The viscosity reduction is more pronounced for the higher $\xi$ value. This is expected since the range of the depletion interaction is broader and thus more free volume can be created to facilitate flow.

The results shown in Figs. 3-5 have been obtained using cone-plate geometry with a gap width at the rim of $1.3 \mathrm{~mm}$. In order to investigate the effect of gap width on the apparent viscosity, we have also done experiments using parallel plate geometry, varying the gap width between 0.1 and $2 \mathrm{~mm}$. Corresponding results are shown in Fig. 6. For the polymer-free dispersion the viscosity data obtained at different gap heights match perfectly over the whole shear rate range covered here. The same is true for the sample with $1 \mathrm{~g} / \mathrm{l}$ polymer added to the continuous phase at gap heights $\geq 0.5 \mathrm{~mm}$. But the apparent low shear viscosity increases by about two orders of magnitude at gap heights between $0.5 \mathrm{~mm}$ and $0.1 \mathrm{~mm}$. The apparent viscosity stays high until a critical shear rate is reached and then drops to the level obtained at high gap heights. This critical shear rate increases with decreasing gap height. This unusual phenomenon is not related to wall slip, instead it indicates clogging of the small gaps and higher shear rates are required to overcome this clogging if the gap width is decreased. The added polymer does not only induce attractive interactions between the particles but also an even stronger attraction between the particles and the wall [Kaplan et al. (1994)] and thus promotes crystallization at the rheometer
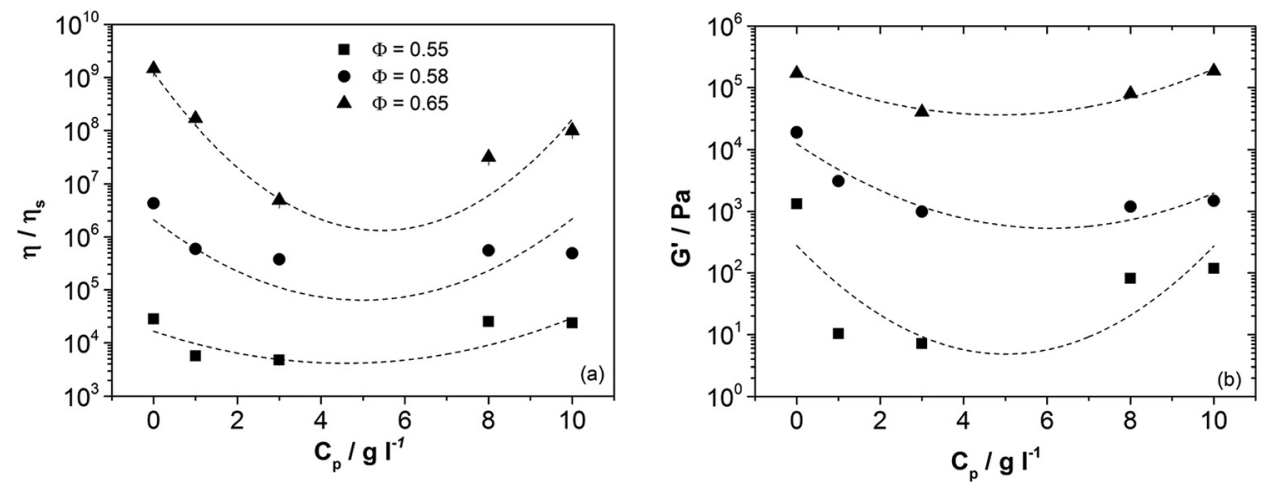

FIG. 4. (a) Relative low shear viscosity determined at $\dot{\gamma}=0.1 \mathrm{~s}^{-1}$ and. (b) Storage modulus $\mathrm{G}^{\prime}$ determined at a constant angular frequency $\omega=1 \mathrm{rad} \mathrm{s}^{-1}$ for different volume fractions as indicated in the legend as a function of added PEO $\left(\mathrm{M}_{\mathrm{w}}=35 \mathrm{~kg} / \mathrm{mol}\right)$. The lines are for visual guidance. 


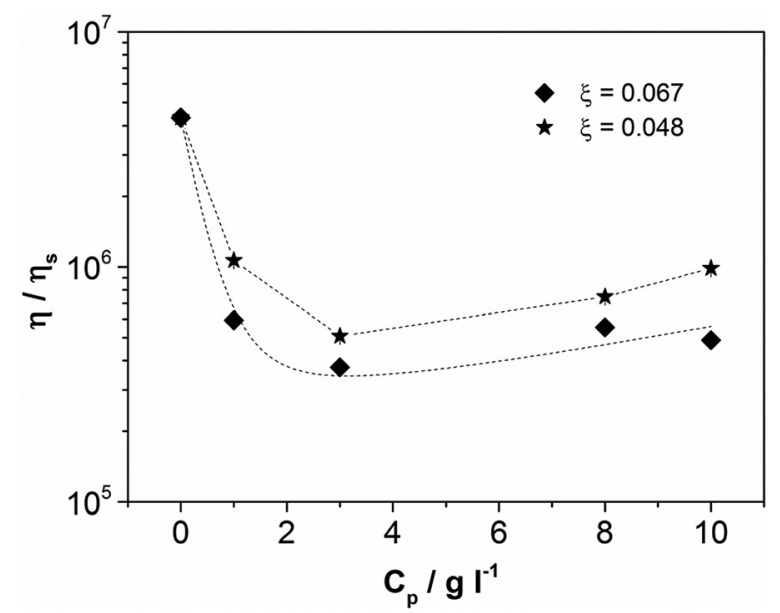

FIG. 5. Low shear viscosity determined a given shear rate $\dot{\gamma}=0.1 \mathrm{~s}^{-1}$ as a function of added PEO concentration for two different molecular weights $\left(\mathrm{M}_{\mathrm{w}}=20\right.$ and $\left.35 \mathrm{~kg} / \mathrm{mol}\right)$ at a constant volume fraction $\Phi=0.58$. The error bars are within the size of the shown symbols. The dotted lines are for visual guidance.

plates. We assume that a crystalline structure builds up spanning the gap between the two parallel plates for the sample with added PEO. This leads at least to a partial clogging of small gaps and to a high apparent viscosity at low shear rates. For the sample without added polymer, this heterogeneous crystallization does not play a significant role.

These observations are confirmed by the flow channel experiments which will be described below.

\section{B. Microscopic flow behavior}

A microchannel with rectangular cross section was used to study the flow behavior of concentrated, crystalline dispersions in microfluidic devices. Glycerin was used as a Newtonian test fluid to validate the particle tracking protocol and to determine the true channel width. As expected, a parabolic flow profile could be retrieved. Good agreement between theoretical and experimentally determined flow velocities was found in these preliminary experiments.

For these channel flow experiments, the particle volume fraction was set to $\Phi=0.52$, at higher particle loadings the viscosity was too high to push the sample through the
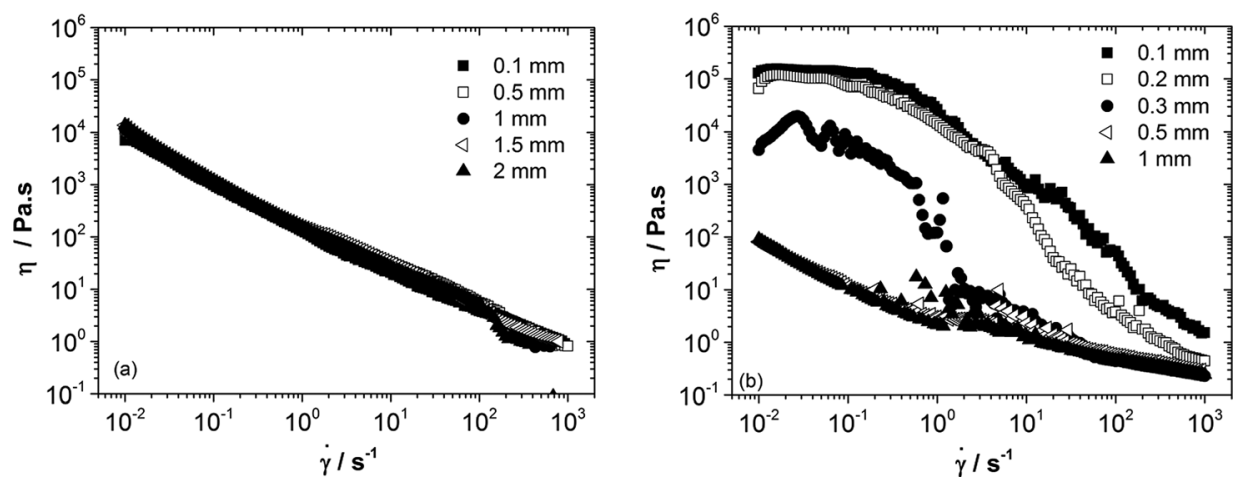

FIG. 6. Apparent viscosity versus shear rate for different gap heights, indicated in the legend for the suspension with $\Phi \approx 0.58$. (a) No added polymer, (b) $1 \mathrm{~g} / \mathrm{l}$ PEO. Rheometer fixture: Plate-plate geometry, $25 \mathrm{~mm}$ diameter. 

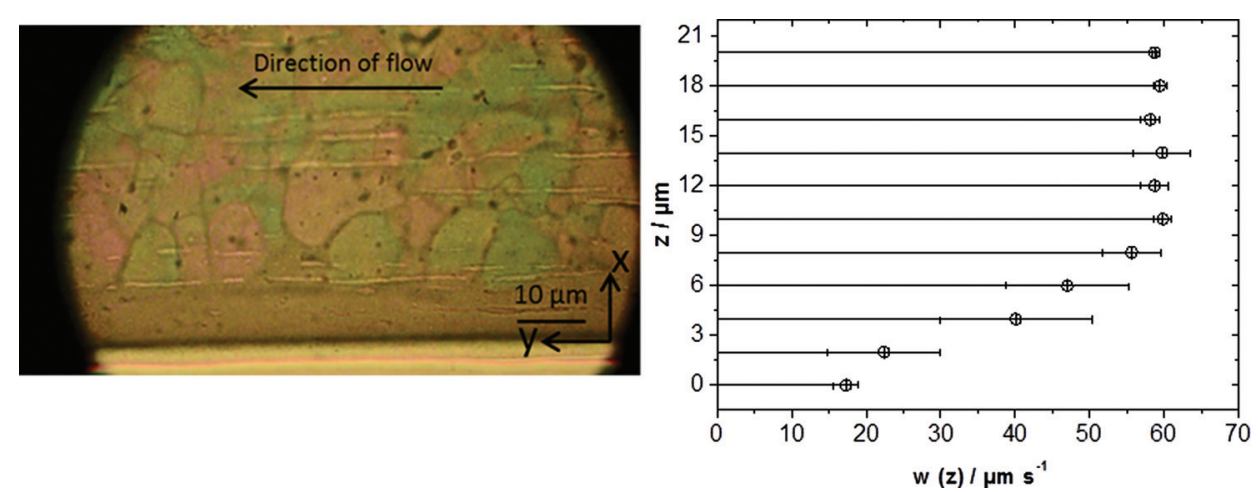

FIG. 7. (a) View through the ocular (x-y plane) on a flow of dispersion without added PEO, $\Phi=0.52$ at shear rate $\dot{\gamma} \approx 20 \mathrm{~s}^{-1}$ (b) corresponding velocity profile in $\mathrm{z}$-direction.

channel with our given setup. PEO molecular mass $\mathrm{M}_{\mathrm{w}}=35 \mathrm{~kg} / \mathrm{mol}$ was selected to achieve a strong viscosity reduction and in addition to the polymer-free suspension two samples with $3 \mathrm{~g} / \mathrm{l}$ and $6 \mathrm{~g} / \mathrm{l}$ added polymer were investigated.

Without added polymer a typical plug flow formed with large crystalline regions in the center of the channel showing up in red and green in Fig. 7(a) and a thin disordered or molten layer next to the wall. The corresponding velocity profile determined in the $\mathrm{z}$ direction perpendicular the $x-y$ (focal) plane is shown in Fig. 7(b). At a nominal wall shear rate $\dot{\gamma}=20 \mathrm{~s}^{-1}$ a slip velocity of about $15 \mu \mathrm{m} \mathrm{s}^{-1}$ at the wall and a constant flow velocity $\mathrm{w} \approx 60 \mu \mathrm{m} \mathrm{s}^{-1}$ in the channel center was revealed. After cessation of flow it took about $10 \mathrm{~min}$ until the whole sample was crystallized and once these crystals touched the channel walls the sample could not be forced to flow anymore. Similar results have been obtained earlier by [Imhof et al. (1994)]. The thickness of the disordered layer decreased with decreasing shear rate and at a nominal wall shear rate $\dot{\gamma} \leq 5 \mathrm{~s}^{-1}$ the shear melting zone vanished and accordingly the channel was clogged.

No steady flow could be established with our setup for the dispersions with added polymer, even though the bulk viscosity was significantly lower than for the polymer-free dispersions. In this cases wall crystallization dominated and the opalescence effect occurred in the syringe and the tubing to the channel immediately after filling. At the inlet of the flow channel big, predominantly red and highly fissured crystals formed at the wall. A large number of smaller crystals were found further downstream. The size of these crystals significantly decreases with increasing PEO concentration as can be seen in Fig. 8.

For the polymer-free system, the average size of crystal domains was about $16 \mu \mathrm{m}$ and decreased to $4 \mu \mathrm{m}$ for the sample including $6 \mathrm{~g} / \mathrm{l}$ polymer. Furthermore, the circularity of the crystals increased with increasing PEO concentration. Further down the flow channel large agglomerates $(>32 \mu \mathrm{m})$ with no defined color or shape occurred. Finally, at the flow front, i.e., at the surface of the dispersion again a large number of crystals were
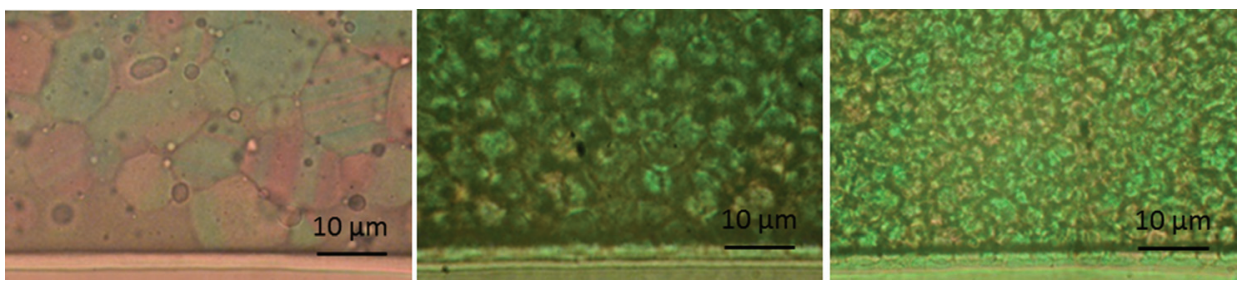

FIG. 8. Crystal size for different amounts of added PEO. Left: No PEO, middle: 3 g/l PEO, right: 6 g/l PEO. 


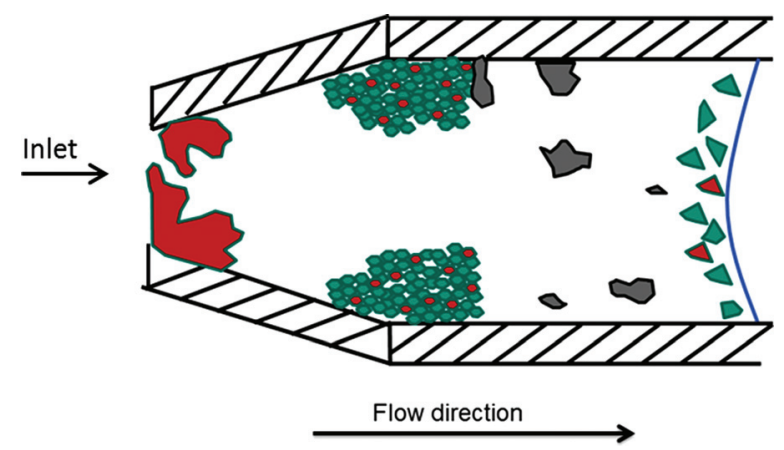

FIG. 9. Different crystallization scenarios in the flow channel for samples with added PEO. At the inlet large crystals which were mainly red, formed with highly fissured surfaces. In the following section of the flow channel a lot of crystals formed with size strongly depending on the concentration of added polymer. Behind that, a region with no crystals but sporadically large agglomerates which showed no defined color extended. At the meniscus of the flow little crystals formed which started to grow once the flow stopped.

observed but in this case their size was found not to depend on polymer concentration. The observed scenario is schematically sketched in Fig. 9. When the flow was stopped due to clogging of the channel inlet, the dispersion started to crystallize from the meniscus toward the channel entrance.

The observations described here are very much in line with the crystallization scenario described by Beyer et al. (2012) who studied the effect of polymer on the crystallization of hard-sphere dispersions via static light scattering. Their investigations revealed a faster induction of wall crystallization as well as a slowing down of precursor to bulk crystal conversion and suppression of bulk crystal growth at higher polymer concentration. This slowing down of bulk crystallization is related to a reduced mobility of colloids due to the added polymer [Zausch et al. (2009); Seefeld and Solomon (2003); Pusey et al. (1993)]. Accordingly, the size of crystals found at the channel inlet in our experiments decreased with increasing polymer concentration. The absence of crystals in the adjacent section of the channel is a consequence of the coexistence of highly concentrated, ordered regions with dilute, isotropic, or fluid areas. This fluid-crystalline coexistence region widens with increasing polymer concentration [Ilett et al. (1995)].

Furthermore, the added polymer not only induces an attractive interaction among particles but also an even stronger attraction between particles and the wall [Dinsmore et al. (1997)]. This promotes wall crystallization which is then dominant in confined geometries like at the inlet of the microchannel used here. This is most likely also the reason for the high apparent viscosity found in rotational rheometry at small gap heights (Fig. 6). Crystals grown from the wall block the gap and the narrower the gap, the more shear energy is required to break up the gap-spanning crystal structure.

\section{SUMMARY AND CONCLUSION}

Introducing weak attractive interactions in crystalline colloidal dispersions leads to a complex flow behavior. We have studied this phenomenon using an electrosterically $\mathrm{P}(\mathrm{S} / \mathrm{BA})$ aqueous polymer dispersion $(\mathrm{R}=130 \mathrm{~nm})$. Weak depletion attraction among particles was introduced by adding the nonadsorbing polymer PEO to the aqueous phase.

The flow properties of this effective hard-sphere system have been studied in steady shear using classical cone-plate and parallel plate rheometry as well as a rectangular microchannel ( $\sim 30 \mu \mathrm{m}$ width) flow cell. 
In macroscopic flow the introduction of weak attraction among particles results in a drastic drop of the low shear viscosity which is attributed the corresponding change of the phase diagram. The addition of nonadsorbing polymer leads to a substantial broadening of the fluid-crystalline coexistence regime and at a given particle loading densely packed crystalline regions coexist with dilute fluid like areas. The density of the crystalline regions increases and the particle loading in the fluid regions decreases with increasing polymer concentration. This result in a reduction of the viscosity, but when a critical polymer concentration is exceeded the strong attractive interaction leads to the formation of a gel-like attractive glass. Accordingly a viscosity minimum is observed. For the system investigated here this minimum occurs around a polymer concentration $\mathrm{c}_{\mathrm{p}} \approx 3 \mathrm{~g} / \mathrm{l}$ well below the overlap concentration $\mathrm{c}_{\mathrm{p}}{ }^{*}$. A minimum polymer to particle size ratio $\xi_{\text {min }}$ is required to induce the viscosity reduction. For the system investigated here $\xi_{\min }=0.03$ and above $\xi_{\min }$, the viscosity reduction increases with increasing $\xi$. A similar size ratio was reported for the fluidization of glass forming systems using nonadsorbing polymer. Finally, in the high shear regime the viscosity is not affected by attractive colloidal interactions since hydrodynamic forces are dominating.

A completely different scenario is observed in microfluidic flow. For the pure colloidal dispersion shear melting is observed close to the channel wall and this enables a pluglike flow of the fluid through the channel. Addition of the polymer slows down bulk crystallization and results in smaller crystals and crystal size decreases with increasing polymer concentration. But at the same time, crystallization at the wall dramatically increases. This leads to a complete clogging of the channel and steady flow conditions cannot be achieved. Wall crystallization also shows up in classical rotational rheometry and results in a strong increase of the apparent viscosity if narrow gap fixtures are used.

The viscosity reduction induced by the added polymer in macroscopic flows might at least qualitatively also be explained by a deswelling of the thin hairy surface layer of the particles investigated here. But this hypothesis fails to explain the observed wall crystallization and corresponding clogging of narrow gaps or flow channels also inferred by the addition of polymer. Both experimental findings can be consistently explained based on the knowledge about the effect of weak depletion attractions on the phase behavior of colloidal hard-sphere dispersions.

As crystallization of proteins is getting more and more important for the production of pharmaceuticals, our observations may help to improve protein preparation. Controlling the size and growth kinetics of protein crystals or fluidization of already crystallized highly concentrated protein solutions could reduce the efforts for the further downstream processing.

\section{ACKNOWLEDGMENTS}

The authors thank BASF SE, especially Spuhler and Dr. Gerst, for donating the styrene-butylacrylate model latex. H. Rehage is thanked for the experiments with the microchannel. The financial support by Deutsche Forschungsgesellschaft DFG Grant No. WI 3138/G3 was gratefully acknowledged.

\section{REFERENCES}

Annunziata, O., O. Ogun, and G. B. Benedek, "Observation of liquid-liquid phase separation for eye lens gScrystallin,” Proc. Natl. Acad. Sci. U.S.A. 100, 970-974 (2003).

Asakura, S., and F. Oosawa, "Interaction between particles suspended in solutions of macromolecules," J. Polym. Sci. 33, 183-192 (1958). 
Ballesta, P., L. Isa, W. C. K. Poon, and R. Besseling, "Wall slip and flow of concentrated hard-sphere colloidal suspensions," J. Rheol. 56, 1005-1037 (2012).

Beyer, R., S. Iacopini, T. Palberg, and H. J. Schöpe, "Polymer induced changes of the crystallization scenario in suspensions of hard sphere like microgel particles,” J. Chem. Phys. 136, 234906 (2012).

Dames, B., B. R. Morrison, and N. Willenbacher, "An empirical model to predict the viscosity of highly concentrated, bimodal dispersions with colloidal interactions," Rheol. Acta. 40, 434-440 (2001).

Dawson, K., G. Foffi, M. Fuchs, W. Götze, F. Sciortino, M. Sperl, P. Tartaglia, Th. Voigtmann, and E. Zaccarelli, "Higher-order glass-transition singularities in colloidal systems with attractive interactions," Phys. Rev. E. 63, 011401 (2001).

Derks, D., Y. L. Wu, A. v. Blaaderena, and A. Imhofa, "Dynamics of colloidal crystals in shear flow," Soft Matter 5, 1060-1065 (2009).

Dijkstra, M., R. v. Roij, and R. Evans, "Phase diagram of highly asymmetric binary hard-sphere mixtures," Phys. Rev. E 59, 5744-5771 (1999).

Dinsmore, A. D., P. B. Warren, W. C. K. Poon, and A. G. Yodh, "Fluid-solid transition on walls in binary hardsphere mixtures," Europhys. Lett. 40(3), 337-342 (1997).

Eckert, T., and E. Bartsch, "Reentrant glass transition in a colloid-polymer mixture with depletion attractions," Phys. Rev Lett. 89, 125701 (2002).

Eckert, T., and E. Bartsch, "The effect of free polymer on interactions and glass transition dynamics of microgel," Faraday Discus. 123, 51-64 (2003).

Eckert, T., and E. Bartsch, "Glass transition dynamics of hard sphere like microgel colloids with short-ranged attractions,” J. Phys. Condens. Matter 16, S4937-S4950 (2004).

Fabbian, L., W. Götze, F. Sciortino, P. Tartaglia, and F. Thiery, "Part A - Rapid Communications-Complex fluids, polymers, and granular materials-ideal glass-glass transitions and logarithmic decay of correlations in a simple system," Phys. Rev. E 59, 2430 (1999).

Fabbian, L., W. Götze, F. Sciortino, P. Tartaglia, and F. Thiery, "Ideal glass-glass transitions and logarithmic decay of correlations in a simple system,” Phys. Rev. E 59, R1347-R1350 (1999).

Farris, R. J., "Prediction of the viscosity of multimodal suspensions from unimodal viscosity data," Trans. Soc. Rheol. 12, 281-301 (1968).

Galkin, O., and P. G. Vekilov, "Control of protein crystal nucleation around the metastable liquid-liquid phase boundary,” Proc. Natl. Acad. Sci. U.S.A. 97, 6277-6281 (2000).

Gast, A. P., C. K. Hall, and W. B. Russel, "Polymer-induced phase separations in nonaqueous colloidal suspensions," J. Colloid Interface Sci. 96, 251-267 (1983).

Hosfield, D., J. Palan, M. Hilgers, D. Scheibe, D. E. McRee, and R. C. Stevens, “A fully integrated protein crystallization platform for small-molecule drug discovery,” J. Struct. Biol. 142, 207-217 (2003).

Ilett, S. M., A. Orrock, W. C. K. Poon, and P. N. Pusey, "Phase behavior of colloid-polymer mixture," Phys. Rev. E. 51(2), 1344-1352 (1995).

Ilett, S. M., W. C. K. Poon, P. N. Pusey, A. Orrock, M. K. Semmler, and S. Erbil, “An experimental study of a model colloid-polymer mixture exhibiting colloidal gas, liquid and crystal phases," in Trends in Colloid and Interface Science VIII, Progress in Colloid \& Polymer Science Volume 97, edited by R. H. Ottewill and A. R. Renniepp (Steinkopff, 1993), pp. 80-84.

Imhof, A., A. van Blaaderen, and J. K. G. Dhont, "Shear melting of colloidal crystals of charged spheres studied with rheology and polarizing microscopy," Langmuir 10, 3477-3484 (1994).

Kalyon, D. M., “Apparent slip and viscoplasticity of concentrated suspensions," J. Rheol. 49, 621-640 (2005).

Kaplan, P. D., J. L. Rouke, and A. G. Yodh, "Entropically driven surface phase separation in binary colloidal mixtures," Phys. Rev. Lett. 72(4), 582-587 (1994).

Kawaguchi, S., G. Imai, J. Suzuki, A. Miyahara, T. Kitano, and K. Ito, “Aqueous solution properties of oligo- and poly(ethylene oxide) by static light scattering and intrinsic viscosity," Polymer 38(12), 2885-2891 (1997).

Kulkarni, A., and C. Zukoski, "Depletion interaction and protein crystallization," J. Cryst. Growth 232, 156-164 (2001).

Lekkerkerker, H. N. W., W. C.-K. Poon, P. N. Pusey, A. Stroobants, and P. B. Warren, "Phase behavior of colloid+polymer mixtures," Europhys. Lett. 20(6), 559-564 (1992). 
Palberg, T., and A. Stipp, "Unusual crystallization kinetics in a hard sphere colloid-polymer mixture," Phys. Rev. Lett. 102, 038302 (2009).

Pandey, R., and J. C. Conrad, "Effects of attraction strength on microchannel flow of colloid-polymer depletion mixtures," Soft Matter 8, 10695-10703 (2012).

Pham, K. N., A. M. Puertas, J. Bergenholtz, S. U. Egelhaaf, A. Moussaï, P. N. Pusey, A. B. Schofield, M. E. Cates, M. Fuchs, and W. C. K. Poon, "Multiple glassy states in a simple model system," Science 296, 104-106 (2002).

Pham, K. N., S. U. Egelhaaf, P. N. Pusey, and W. C. K. Poon, "Glasses in hard spheres with short-range attraction,” Phys. Rev. E. 69, 011503 (2004).

Poon, W. C. K., "The physics of a model colloid-polymer mixture," J. Phys.: Condens. Matter 14, R859-R880 (2002).

Pusey, P. N., A. D. Pirie, and W. C. K. Poon, "Dynamics of colloid-polymer mixtures," Physica A 201, 322-331 (1993).

Pusey, P. N., W. C. K. Poon, S. M. Ilett, and P. Bartlett, "Phase behavior and structure of colloidal suspensions," J. Phys.: Condens Matter 6, A29-A36 (1994).

Pusey, P. N., and W. van Megen, "Phase behavior of concentrated suspensions of nearly hard colloidal spheres," Nature 320, 340-342 (1986).

Pusey, P. N., W. Van Megen, S. M. Underwood, P. Bartlett, and R. H. Ottewill, "Colloidal fluids, crystals and glasses," Physica A 176, 16-27 (1991).

Seefeld, K. F., and M. J. Solomon, "Self diffusion in dilute colloidal suspensions with attractive potential interactions,” Phys. Rev. E 67, 050402 (2003).

Segré, P. N., O. P. Behrend, and P. N. Pusey, "Short-time Brownian motion in colloidal suspensions: Experiments and simulation," Phys. Rev. E 52, 5070-5083 (1995).

Shah, S. A., Y.-L. Chen, K. S. Schweizer, and C. F. Zukoski, "Phase behavior and concentration fluctuations in suspensions of hard spheres and nearly ideal polymers," J. Chem. Phys. 118, 3350-3361 (2003a).

Shah, S. A., Y.-L. Chen, K. S. Schweizer, and C. F. Zukoski, "Viscoelasticity and rheology of depletion flocculated gels and fluids," J. Chem. Phys. 119, 8747-8760 (2003b).

Smith, P. A., G. Petekidis, S. U. Egelhaaf, and W. C. K. Poon, "Yielding and crystallization of colloidal gels under oscillatory shear," Phys. Rev. E 76, 041402 (2007).

Sollich, P., and N. B. Wilding, "Polydispersity induced solid-solid transitions in model colloids," Soft Matter 7, 4472-4484 (2011).

Stipp, A., H.-J. Schöpe, T. Palberg, T. Eckert, R. Biehl, and E. Bartsch, "Optical experiments on a crystallizing hard-sphere-polymer mixture at coexistence,” Phys. Rev. E 81(5), 051401 (2010).

Valadez-Pérez, N. E., A. L. Benavides, E. Schöll-Paschinger, and R. Castañeda-Priego, "Phase behavior of colloids and proteins in aqueous suspensions: Theory and computer simulations," J. Chem. Phys. 137, 084905 (2012).

Vesaratchanon, J. S., K. Takamura, and N. Willenbacher, "Surface characterization of functionalized latexes with different surface functionalities using rheometry and dynamic light scattering," J. Colloid Interface Sci. 345, 214-221 (2010).

Walls, H. J., S. B. Caines, A. M. Sanchez, and S. A. Khan, "Yield stress and wall slip phenomena in colloidal," J. Rheol. 47, 847-868 (2003).

Willenbacher, N., J. S. Vesaratchanon, O. Thorwarthb, and E. Bartschb, “An alternative route to highly concentrated, freely flowing colloidal dispersions," Soft Matter 7, 5777-5788 (2011).

Wyss, H. M., D. L. Blair, J. F. Morris, H. A. Stone, and D. A. Weitz, "Mechanism for clogging of microchannels," Phys. Rev. E 74, 061402 (2006).

Zaccarelli, E., F. Sciortino, and P. Tartaglia, "Numerical study of the glass-glass transition in short-ranged attractive colloids,” J. Phys.: Condens. Matter 16, S4849-S4860 (2004).

Zausch, J., P. Virnau, K. Binder, J. Horbach, and R. L. Vink, "Statics and dynamics of colloid-polymer mixtures near their critical point of phase separation: A computer simulation study of a continuous Asakura-Oosawa model,” J. Chem. Phys. 130, 064906 (2009). 